\title{
Thermodynamical Analysis of the Flow and Heat Transfer over a Static and a Moving Wedge
}

\author{
Adnan Saeed Butt and Asif Ali \\ Department of Mathematics, Quaid-I-Azam University, Islamabad 45320, Pakistan \\ Correspondence should be addressed to Adnan Saeed Butt; adnansaeedbutt85@gmail.com
}

Received 12 August 2013; Accepted 24 September 2013

Academic Editors: G. L. Aranovich, A. Chagovetz, and M. Sanati

Copyright ( 2013 A. S. Butt and A. Ali. This is an open access article distributed under the Creative Commons Attribution License, which permits unrestricted use, distribution, and reproduction in any medium, provided the original work is properly cited.

\begin{abstract}
The first and second law characteristics of fluid flow and heat transfer over a static and a moving wedge are investigated. With the help of suitable similarity transformations, the governing boundary layer equations for the velocity and temperature fields are transformed into ordinary differential equations and are solved numerically. The velocity and the temperature profiles are obtained for various parameters and are utilized to compute the entropy generation number Ns and the Bejan number Be. The effects of various physical parameters on the entropy generation number and the Bejan number are depicted through graphs and are discussed qualitatively. It is observed that the entropy production rate is less in case of wedge moving in the opposite direction to flow as compared to static wedge.
\end{abstract}

\section{Introduction}

Newton's second law of motion and laws of thermodynamics are the fundamental principles on which all the flow and heat transfer systems are built today. All other laws play a supportive role. First law of thermodynamics gives information about the energy of the system quantitatively. On the other hand, second law of thermodynamics states that all real life processes are irreversible. The irreversibility of the processes is measured by entropy generation. The pioneer work on entropy generation in flow systems was done by Bejan $[1,2]$. He showed that the engineering design of a thermal system can be improved by minimizing the entropy generation. Later on, a lot of research has been done by many other investigators by taking different geometrical configurations related to the thermally designed systems and fluid flow processes. Sahin [3] analyzed the entropy effects in viscous fluid flow in a circular duct with the isothermal boundary condition. The first and second law characteristics of fluid flow and heat transfer in a channel having two parallel plates with a finite gap between them were analytically investigated by Mahmud and Fraser [4]. Yilbas et al. [5] discussed the entropy production effects in a semiblocked pipe by taking into consideration the swirling effects. The influence of magnetic field on entropy generation in laminar forced flow past a horizontal flat plate was examined by Al-Odat et al. [6]. Selamet and Arpaci [7] investigated the entropy effects in boundary layer flows. Makinde and Osalusi [8] studied entropy effects in a liquid film falling along an inclined heated porous flat plate. Makinde [9] extended his work and analyzed the irreversibility effects in nonNewtonian liquid film falling under the influence of gravity force. The entropy generation effects in the slip MHD flow in a single rotating disk were studied by Arikoglu et al. [10]. Makinde [11] discussed the irreversibility effects in variable viscosity channel flow with convective cooling at the walls. Second law analysis for a gravity driven variable viscosity liquid film along an inclined heated plate with convective cooling was made by Makinde [12]. Révèillere and Baytaş [13] studied entropy generation in laminar boundary layer flow over an isothermal permeable flat surface. Makinde and Bég [14] used perturbation technique together with HermitePade approximation to examine the entropy generation in a reactive hydromagnetic channel flow. The first and second law characteristics of fluid flow in a porous medium over a permeable stretching surface were investigated by Tamayol et al. [15]. The thermodynamical analysis of MHD boundary layer flow over a flat plate with variable viscosity was done by Makinde [16]. He considered that the thermal radiation 


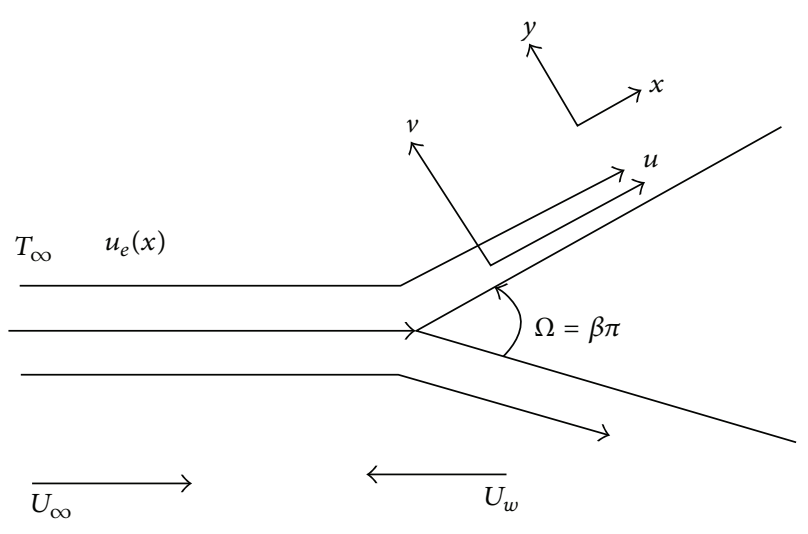

FIGURE 1: Schematic diagram of the problem.

is also present there, and the surface is convectively heated. It was found that the variable viscosity results in more entropy production. Makinde and Aziz [17] discussed the entropy generation effects in a long hollow cylinder with time dependent internal heating and convective heating at both inner and outer surfaces. Analytical solution of the problem was obtained, and a thermal stability criterion was also examined. Chen et al. [18] made use of differential transform method to study the entropy effects in the mixed convective flow in a vertical channel. Butt et al. [19] examined the viscoelastic effects on the entropy generation in fluid flow and heat transfer through a porous medium over a permeable stretching surface. The study of entropy production in MHD slip flow over a vertical surface with convective boundary was done by Butt et al. [20]. Munawar and Ali [21] examined the entropy generation in fluid flow and heat transfer over an oscillating stretching cylinder. The effects of magnetic field on entropy generation in flow over a radially stretching sheet were investigated by Butt and Ali [22].

\section{Mathematical Formulation of the Problem}

Consider a steady state two-dimensional boundary layer flow of a laminar incompressible fluid past a static and a moving wedge as shown in the Figure 1. The coordinate system is chosen such that the $x$-coordinate is parallel to the surface of the wedge and the $y$-coordinate is normal to it. The properties of the fluid are assumed to be constant. It is assumed that the velocity of the free stream (inviscid flow) is $u_{e}(x)=U_{\infty} x^{m}$ and that of the moving wedge is $u_{w}(x)=U_{w} x^{m}$, where $U_{\infty}$, $U_{w}$, and $m$ are constants with $0 \leq m \leq 1$. Using the boundary layer approximations, the governing equations for the steady flow and heat transfer over a wedge are given by

$$
\begin{gathered}
\frac{\partial u}{\partial x}+\frac{\partial v}{\partial y}=0 \\
u \frac{\partial u}{\partial x}+v \frac{\partial u}{\partial y}=v \frac{\partial^{2} u}{\partial y^{2}}+u_{e} \frac{d u_{e}}{d x} \\
u \frac{\partial T}{\partial x}+v \frac{\partial T}{\partial y}=\alpha \frac{\partial^{2} T}{\partial y^{2}}+\frac{\nu}{\rho c_{p}}\left(\frac{\partial u}{\partial y}\right)^{2}
\end{gathered}
$$

The corresponding boundary conditions for the flow are

(i) static wedge:

$$
\begin{aligned}
& u=0, \quad v=0 \quad \text { at } y=0, \\
& u=u_{e}(x) \quad \text { at } y \longrightarrow \infty,
\end{aligned}
$$

(ii) moving wedge:

$$
\begin{aligned}
& u=u_{w}(x), \quad v=0 \quad \text { at } y=0, \\
& u=u_{e}(x) \quad \text { at } y \longrightarrow \infty .
\end{aligned}
$$

The thermal boundary conditions are

$$
T=T_{w} \quad \text { at } y=0, \quad T=T_{\infty} \quad \text { at } y \longrightarrow \infty .
$$

Here $u$ and $v$ are the velocity components along the $x$ and $y$ axes, respectively, $v$ is the kinematic viscosity, $\alpha$ is the thermal diffusivity, $\rho$ is the density of the fluid, $c_{p}$ is the specific heat of the fluid at a constant pressure, $T$ is the temperature of the fluid, and $T_{w}$ is the wall temperature, $T_{\infty}$ is the temperature far away from the surface.

Introducing the similarity transformation, we have

$$
\begin{aligned}
& \psi(x, y)=\sqrt{\frac{2 v x u_{e}(x)}{m+1} f(\eta),} \\
& \eta(x, y)=\sqrt{\frac{(m+1) u_{e}(x)}{2 v x}} y,
\end{aligned}
$$

where $\psi$ is the stream function defined such that $u=$ $\partial \psi / \partial y, v=-\partial \psi / \partial x$ satisfy (1) identically. The similarity transformation for temperature is

$$
\theta=\frac{T-T_{\infty}}{T_{w}-T_{\infty}} .
$$

Substituting (7) and (8) into (1)-(6), we have

$$
\begin{gathered}
f^{\prime \prime \prime}+f f^{\prime \prime}+\frac{2 m}{m+1}\left(1-f^{\prime 2}\right)=0 \\
\theta^{\prime \prime}+\operatorname{Pr} f \theta^{\prime}+\operatorname{PrEc} f^{\prime \prime 2}=0 .
\end{gathered}
$$

The boundary conditions for flow are

(i) static wedge:

$$
f(0)=0, \quad f^{\prime}(0)=0, \quad f^{\prime}(\infty)=1,
$$

(ii) moving wedge:

$$
f(0)=0, \quad f^{\prime}(0)=\lambda, \quad f^{\prime}(\infty)=1 .
$$

The boundary conditions for temperature in nondimensional form are

$$
\theta(0)=1, \quad \theta(\infty)=0,
$$


where prime denotes the differentiation with respect to $\eta$, $\operatorname{Pr}=\mu c_{p} / k$ is the Prandtl number, Ec $=U^{2} / c_{p}\left(T_{w}-T_{\infty}\right)$ is the Eckert number, and the parameters $\lambda$ and $\beta$ are defined as

$$
\lambda=\frac{U_{w}}{U_{\infty}}, \quad \beta=\frac{2 m}{m+1} .
$$

Here $\lambda$ is the moving wedge parameter where $\lambda>0$ corresponds to the motion of wedge in the direction of free stream and $\lambda<0$ corresponds to a moving wedge in the opposite direction of the free stream velocity. The case $\lambda=0$ corresponds to a static wedge.

\section{Entropy Generation}

According to Bejan $[2,23]$, the entropy generation per unit volume is defined as

$$
S_{G}=\frac{k}{T^{2}}\left[\left(\frac{\partial T}{\partial x}\right)^{2}+\left(\frac{\partial T}{\partial y}\right)^{2}\right]+\frac{\mu}{T}\left(\frac{\partial u}{\partial y}\right)^{2} .
$$

The first term in (14) is the entropy generation due to heat transfer, and the second term is the entropy generation due to viscous dissipation. The dimensionless form of the entropy generation is given by

$$
\begin{aligned}
\mathrm{Ns}= & \frac{S_{G}}{S_{o}} \\
= & \left(\left(\frac{m-1}{2}\right)^{2} \eta^{2}+\left(\frac{m+1}{2}\right) \operatorname{Re}_{x}\right) \theta^{\prime 2} \\
& +\frac{\mathrm{Br}}{\Omega}\left(\frac{m+1}{2}\right) \operatorname{Re}_{x} f^{\prime \prime 2},
\end{aligned}
$$

where $S_{o}=k\left(T_{w}-T_{\infty}\right)^{2} / T_{w}{ }^{2} x^{2}$ is the characteristic entropy generation rate, $\Omega=\left(T_{w}-T_{\infty}\right) / T_{w}$ is the dimensionless temperature difference, $\operatorname{Re}_{x}=U x / \nu$ is the local Reynolds number, and $\mathrm{Br}=\mathrm{PrEc}$ is the Brinkman number.

An alternative irreversibility distribution parameter is the Bejan number, which gives an idea whether the fluid friction irreversibility dominates over heat transfer irreversibility or the heat transfer irreversibility dominates over fluid friction rreversibility. It is simply the ratio of entropy generation due to heat transfer to the total entropy generation:

$$
\mathrm{Be}=\frac{\text { Entropy generation due to heat transfer }}{\text { Total entropy generation }} \text {. }
$$

When $\mathrm{Be} \gg 0.5$, the irreversibility due to heat transfer dominates, whereas when $\mathrm{Be} \ll 0.5$, the irreversibility due to viscous effects dominates. When $\mathrm{Be}=0.5$, the heat transfer and the fluid friction irreversibilities are equal.

\section{Results and Discussion}

The nonlinear differential equation (9) with boundary conditions (10)-(12) is solved using the shooting method with Runge-Kutta algorithm. A step size of 0.001 is taken to obtain

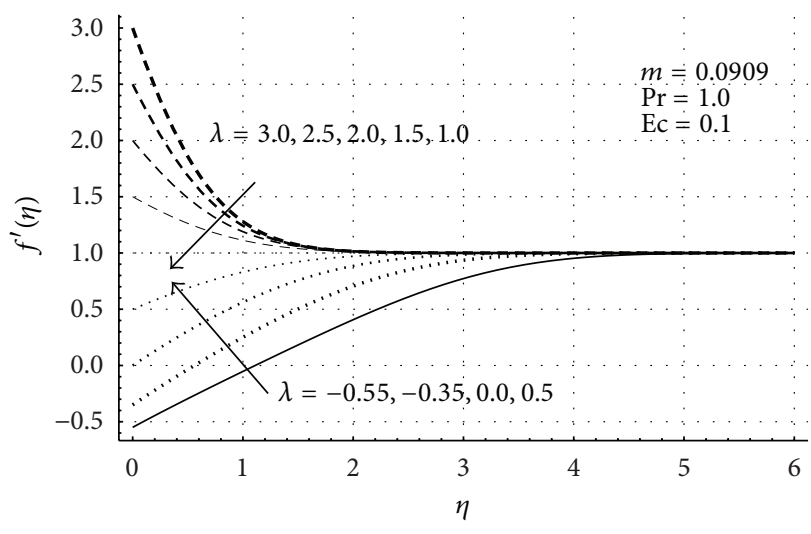

FiguRE 2: Effects of moving wedge parameter $\lambda$ on $f^{\prime}(\eta)$.

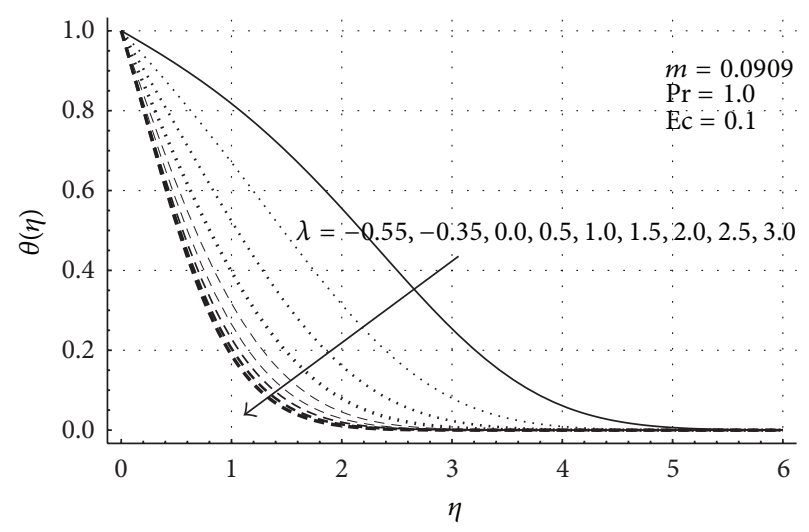

FIGURE 3: Effects of moving wedge parameter $\lambda$ on $\theta(\eta)$.

the numerical solution, and the accuracy goal is kept equal to $10^{-7}$. The effects of different physical parameters on velocity and temperature profiles are analyzed by plotting graphs. Moreover, the graphs of the entropy generation number and the Bejan number are presented to examine the entropy production in the considered problem.

Figure 2 illustrates that for nonpositive values of $\lambda$; that is, when the wedge is moving in the opposite direction of stream velocity, the momentum boundary layer thickness is more than the case when both wedge and stream are moving in the same direction $\lambda \geq 0$. Moreover $\lambda=1$ corresponds to the case when the fluid and the wedge move with same velocities. In this situation, there is no shear stress at the surface. Figure 3 depicts the effects of moving wedge parameter $\lambda$ on temperature profile. It is observed that the thermal boundary layer thickness decreases all values of $\lambda$. The influence of Prandtl number Pr on temperature profile is presented in Figure 4. It is observed that with an increase in the Prandtl number, the thermal diffusion rate decreases which ultimately results in decreasing of the thermal boundary layer thickness. The effects of Eckert number Ec on temperature profile are shown in Figure 5. An increase in thermal boundary layer is noticed with an increase in the value of Ec.

The variations in entropy generation number Ns are plotted against $\eta$ in Figures 6, 7, 8, and 9 for different 


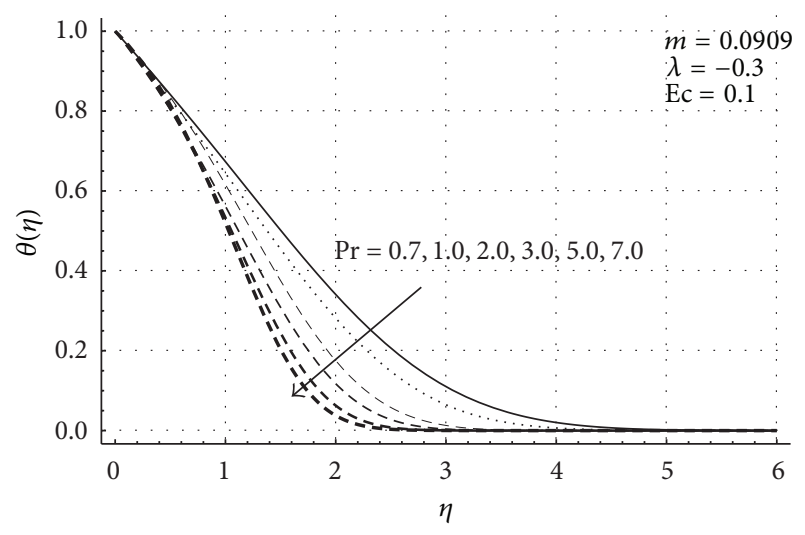

Figure 4: Effects of Prandtl number on temperature profile $\theta(\eta)$.

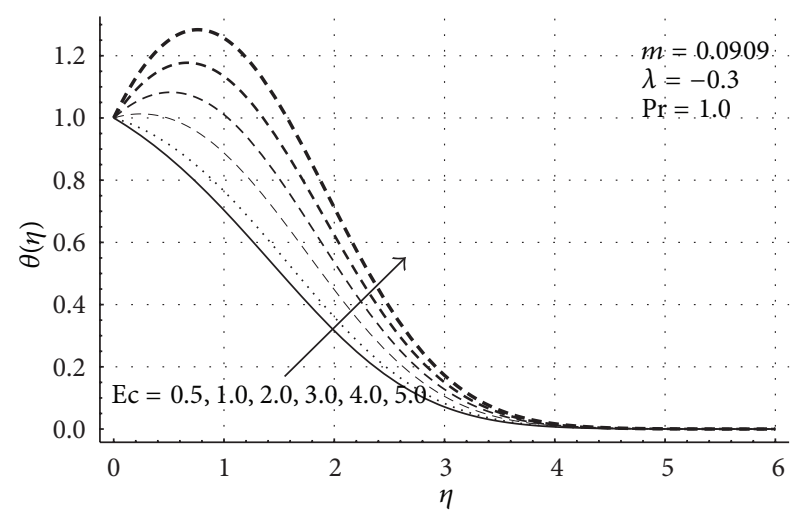

FIGURE 5: Effects of Eckert number on temperature profile $\theta(\eta)$.

physical parameters. Figure 6 shows the effects of Prandtl number Pr on entropy profile. For a particular value of Pr entropy generation increases and attains its peak value within the boundary layer region. Then it decreases sharply and becomes zero in the free stream flow region. Also it is noteworthy that the irreversibility effects are observed within a small region of boundary layer for higher values of Prandtl number as compared to the small Prandtl number. The effects of moving wedge parameter $\lambda$ on Ns are shown in Figure 7. It is noticed from the figure that near the surface entropy production increases by increasing $\lambda$ and an opposite behavior is observed far away from the surface. Moreover, in the case when wedge and free stream are moving oppositely, that is, $\lambda<0$, the entropy effects can be observed for large distance within the boundary layer region. The effects of the group parameter $\mathrm{Br} / \Omega$ on entropy generation number Ns are shown in Figure 8. Increasing the value of $\mathrm{Br} / \Omega$ results in more entropy production. It is also observed that for a particular value of $\mathrm{Br} / \Omega$, the entropy generation increases with an increase in $\eta$ and attains its maximum value at $\eta=1.7$ (roughly) and then decreases and approaches zero as the free stream region is reached. The influence of the local Reynolds number $\mathrm{Re}_{x}$ on Ns is depicted from Figure 9. It can be seen that the entropy production increases as $\mathrm{Re}_{x}$ increases.

The Bejan number Be is plotted in Figures 10-13 for different ranges of various parameters. Figure 10 depicts that,

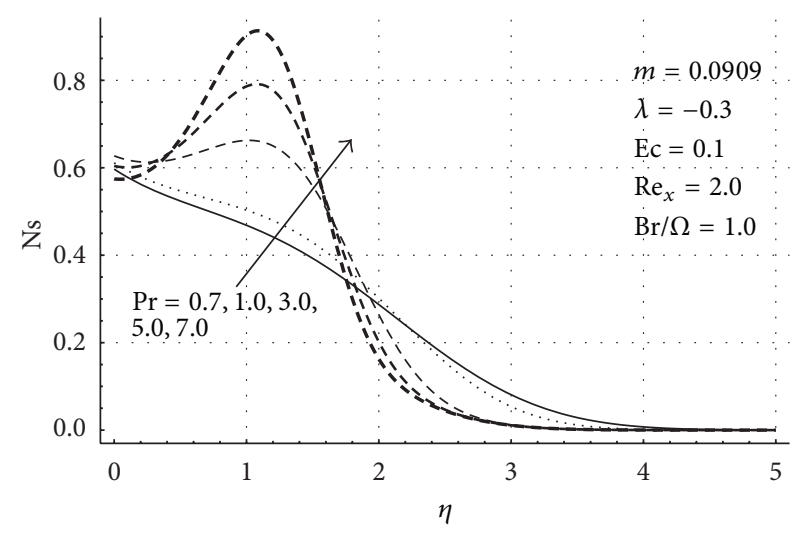

Figure 6: Effects of Prandtl number Pr on entropy generation number Ns.

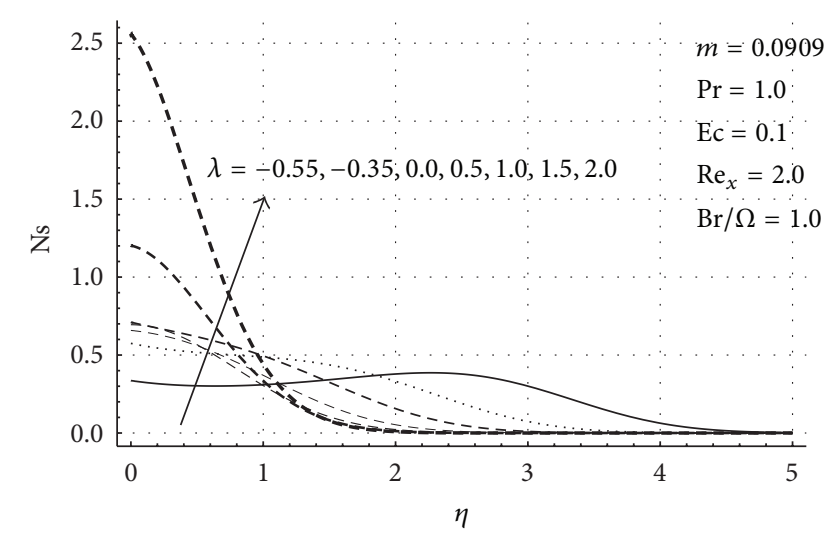

FIGURE 7: Effects of moving wedge parameter $\lambda$ on entropy generation number Ns.

for small values of the Prandtl number Pr, the irreversibility effects due to viscous dissipation are dominant near the wedge surface, and afterward heat transfer irreversibility becomes prominent. However, for large values of $\mathrm{Pr}$, the scenario is quite different. It is noticed that, at the surface, irreversibility due to viscous dissipation is dominant, and then the heat transfer irreversibility becomes strong in the boundary layer region by increasing Pr. In the main stream region entropy production due to viscous dissipation effects again become strong as $\mathrm{Pr}$ increases. Figure 11 shows the behavior of the Bejan number for various values of moving wedge parameter $\lambda$. It is quite evident that, by increasing $\lambda$ in the range $-0.55 \leq \lambda \leq 1$ the entropy effects due to heat transfer become strong. For $\lambda>1$, an opposite trend is noticed, and the entropy due to fluid friction is dominant. This means that irreversibility due to viscous effects remains strong when wedge moves in the opposite direction to the free stream velocity or when the velocity of the wedge is greater than the velocity of free stream flow. Obviously, this conduct is due to large frictional forces generated near the surface in both cases. Figure 12 illustrates that the fluid friction irreversibility becomes dominant by increasing the group parameter $\mathrm{Br} / \Omega$ at the surface. It is quite obvious that this behavior is due to the increasing effects of viscous 


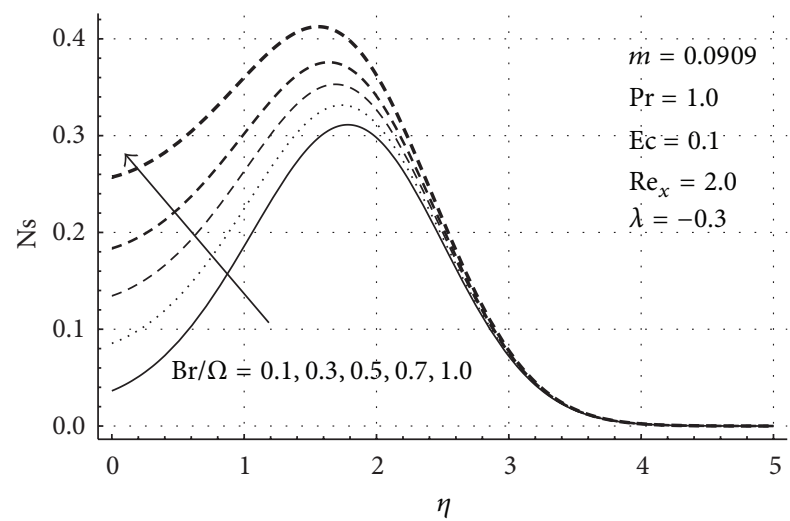

FIGURE 8: Effects of group parameter $\mathrm{Br} / \Omega$ on entropy generation number Ns.

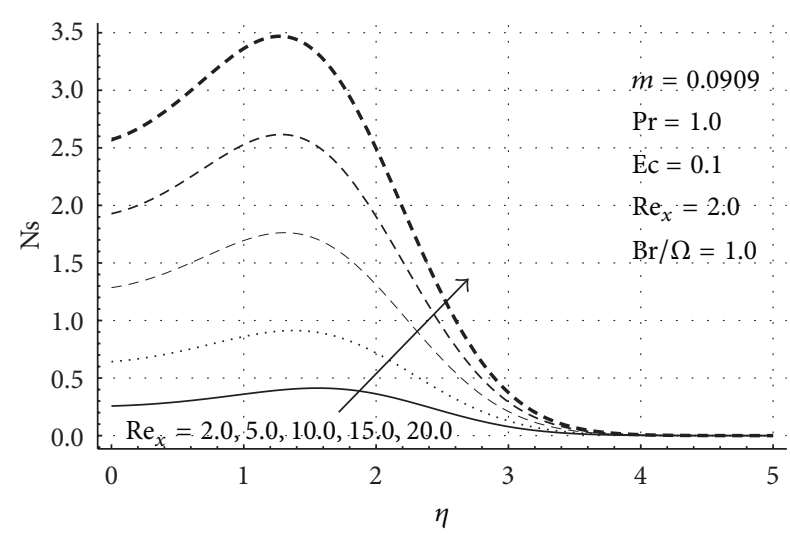

FIGURE 9: Effects of local Reynolds number $\mathrm{Re}_{x}$ on entropy generation number Ns.

dissipation in the flow. In Figure 13, the influence of local Reynolds number $\operatorname{Re}_{x}$ on Bejan number is presented. The heat transfer irreversibility becomes weak as $\mathrm{Re}_{x}$ increases. However, these effects are not significant near the surface.

\section{Conclusion}

The second law (of thermodynamics) characteristics of viscous incompressible fluid flow over a static and moving wedge are investigated. The velocity and the temperature fields are used to obtain the expression for the entropy generation. In order to make a comparison whether the heat transfer irreversibility dominates over the viscous dissipation irreversibility or vice versa, the Bejan number is plotted for different physical parameter, and the results are discussed briefly. It is noticed that when the wedge and fluid are moving in opposite directions $(\lambda<0)$, the momentum boundary layer is thicker than the case when the fluid and the wedge are moving in same direction. However, the thermal boundary layer thickness decreases for both moving and static wedge. It is concluded that the fluids with large Pr have small thermal boundary layers. Moreover, the thermal boundary layer thickens by increasing the Eckert number Ec. The effects of Prandtl number Pr on entropy generation number are

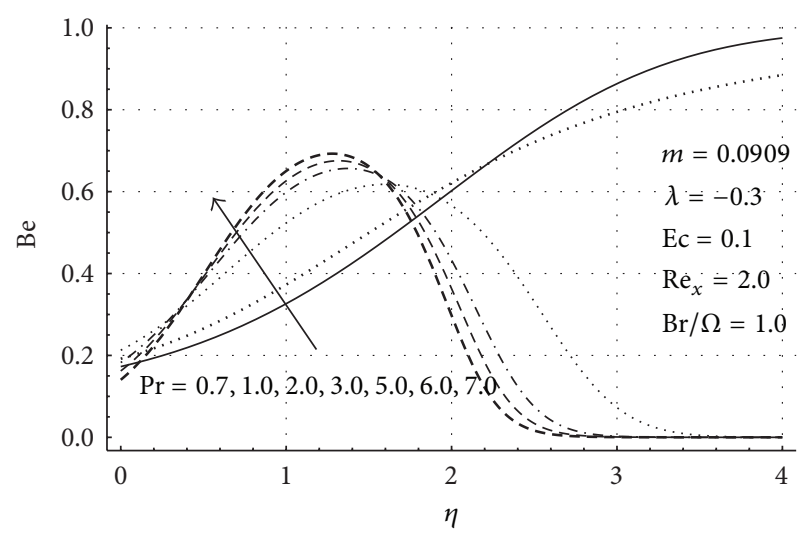

Figure 10: Effects of Prandtl number Pr on the Bejan number Be.

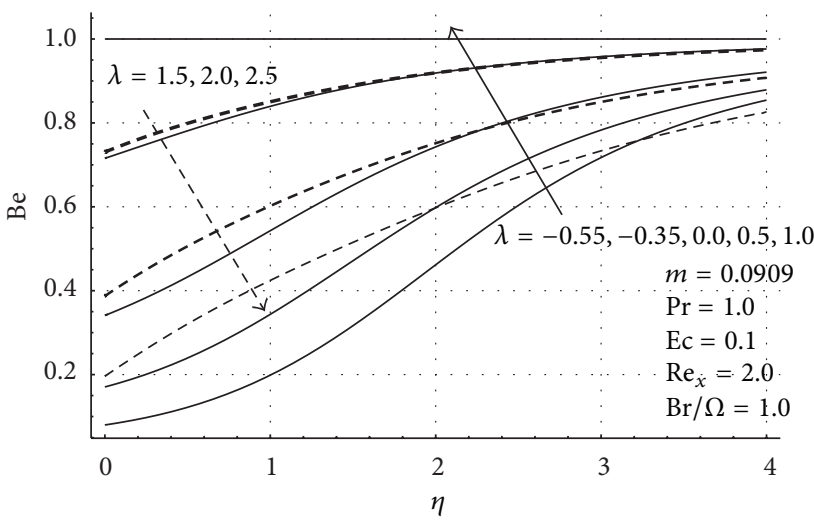

FIGURE 11: Effects of moving wedge parameter $\lambda$ on the Bejan number Be.

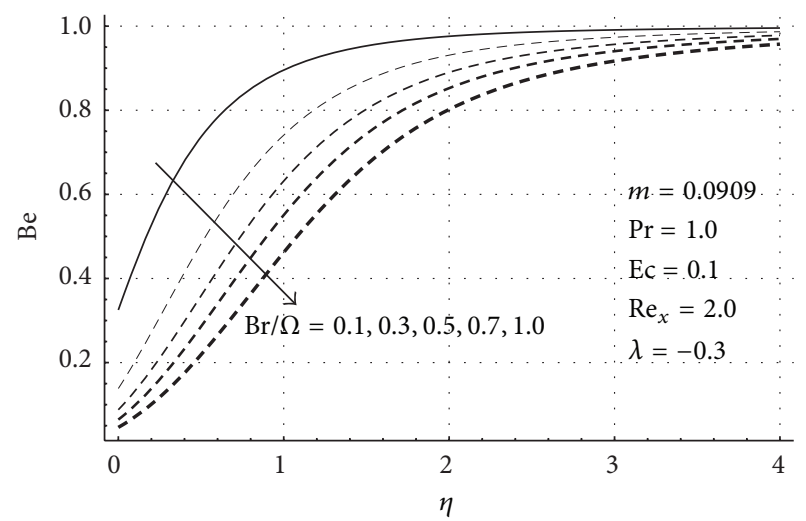

FIGURE 12: Effects of group parameter $\mathrm{Br} / \Omega$ on the Bejan number Be.

increasing within the boundary layer region. However, for large values of $\mathrm{Pr}$, the irreversibility effects are observed in a small region. The entropy generation increases as $\lambda$ increases near the surface and decreases far away from the surface. Moreover, it can be seen that the entropy generation is minimum when wedge moves in the opposite direction of free stream velocity. The presence of the group parameter $\mathrm{Br} / \Omega$ and the local Reynolds number $\mathrm{Re}_{x}$ is to increase 


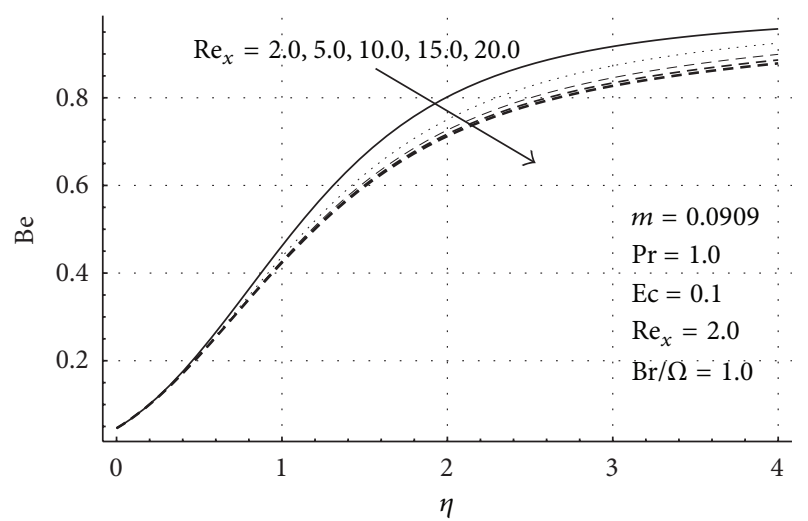

Figure 13: Effects of local Reynolds number $\operatorname{Re}_{x}$ on the Bejan number Be.

entropy production. It is observed from the analysis that fluid friction irreversibility dominates when the wedge is moving in the opposite direction of free stream velocity or when the wedge is moving quickly as compared to the free stream velocity. The fluid friction irreversibility effects become stronger as the group parameter $\mathrm{Br} / \Omega$ and the local Reynolds number $\mathrm{Re}_{x}$ increase.

\section{References}

[1] A. Bejan, "A study of entropy generation in fundamental convective heat transfer," Journal of Heat Transfer, vol. 101, no. 4, pp. 718-725, 1979.

[2] A. Bejan, Entropy Generation Through Heat and Fluid Flow, John Wiley and Sons, Toronto, Canada, 1994.

[3] A. Z. Sahin, "Second law analysis of laminar viscous flow through a duct subjected to constant wall temperature," Journal of Heat Transfer, vol. 120, pp. 76-83, 1998.

[4] S. Mahmud and R. A. Fraser, "Thermodynamic analysis of flow and heat transfer inside channel with two parallel plates," Exergy, vol. 2, pp. 140-146, 2002.

[5] B. S. Yilbas, S. Z. Shuja, and M. O. Budair, "Second law analysis of a swirling flow in a circular duct with restriction," International Journal of Heat and Mass Transfer, vol. 42, no. 21, pp. 4027-4041, 1999.

[6] M. Q. Al-Odat, R. A. Damseh, and M. A. Al-Nimr, "Effect of magnetic field on entropy generation due to laminar forced convection past a horizontal flat plate," Entropy, vol. 6, no. 3, pp. 293-303, 2004.

[7] A. Selamet and V. S. Arpaci, "Entropy production in boundary layers," Journal of Thermophysics and Heat Transfer, vol. 4, no. 3, pp. 404-407, 1990.

[8] O. D. Makinde and E. Osalusi, "Entropy generation in a liquid film falling along an inclined porous heated plate," Mechanics Research Communications, vol. 33, no. 5, pp. 692-698, 2006.

[9] O. D. Makinde, "Irreversibility analysis for a gravity driven non-Newtonian liquid film along an inclined isothermal plate," Physica Scripta, vol. 74, no. 6, article 007, pp. 642-645, 2006.

[10] A. Arikoglu, I. Ozkol, and G. Komurgoz, "Effect of slip on entropy generation in a single rotating disk in MHD flow," Applied Energy, vol. 85, no. 12, pp. 1225-1236, 2008.
[11] O. D. Makinde, "Irreversibility analysis of variable viscosity channel flow with convective cooling at the walls," Canadian Journal of Physics, vol. 86, no. 2, pp. 383-389, 2008.

[12] O. D. Makinde, "Thermodynamic second law analysis for a gravity-driven variable viscosity liquid film along an inclined heated plate with convective cooling," Journal of Mechanical Science and Technology, vol. 24, no. 4, pp. 899-908, 2010.

[13] A. Réveillère and A. C. Baytaş, "Minimum entropy generation for laminar boundary layer flow over a permeable plate," International Journal of Exergy, vol. 7, no. 2, pp. 164-177, 2010.

[14] O. D. Makinde and O. A. Bég, "On inherent irreversibility in a reactive hydromagnetic channel flow," Journal of Thermal Science, vol. 19, no. 1, pp. 72-79, 2010.

[15] A. Tamayol, K. Hooman, and M. Bahrami, "Thermal analysis of flow in a porous medium over a permeable stretching wall," Transport in Porous Media, vol. 85, no. 3, pp. 661-676, 2010.

[16] O. D. Makinde, "Second law analysis for variable viscosity hydromagnetic boundary layer flow with thermal radiation and Newtonian heating," Entropy, vol. 13, no. 8, pp. 1446-1464, 2011.

[17] O. D. Makinde and A. Aziz, "Analysis of entropy generation and thermal stability in a long hollow cylinder with asymmetry convective cooling," Heat and Mass Transfer, vol. 47, no. 11, pp. 1407-1415, 2011.

[18] C.-K. Chen, H.-Y. Lai, and C.-C. Liu, "Numerical analysis of entropy generation in mixed convection flow with viscous dissipation effects in vertical channel," International Communications in Heat and Mass Transfer, vol. 38, no. 3, pp. 285-290, 2011.

[19] A. S. Butt, S. Munawar, A. Ali, and A. Mehmood, "Effect of viscoelasticity on entropy generation in a porous medium over a stretching plate," World Applied Sciences Journal, vol. 17, no. 4, pp. 516-523, 2012.

[20] A. S. Butt, S. Munawar, A. Ali, and A. Mehmood, "Entropy generation in hydrodynamic slip flow over a vertical plate with convective boundary," Journal of Mechanical Science and Technology, vol. 26, no. 9, pp. 2977-2984, 2012.

[21] A. S. Butt and A. Ali, "Effects of magnetic field on entropy generation in flow and heat transfer due to radially stretching surface," Chinese Physics Letters, vol. 30, no. 2, pp. 02704-02708, 2012.

[22] S. Munawar and M. A. Ali, "Thermal analysis of the flow over an oscillatory stretching cylinder," Physica Scripta, vol. 86, Article ID 065401, 2012.

[23] L. C. Woods, Thermodynamics of Fluid Systems, Oxford University Press, Oxford, UK, 1975. 

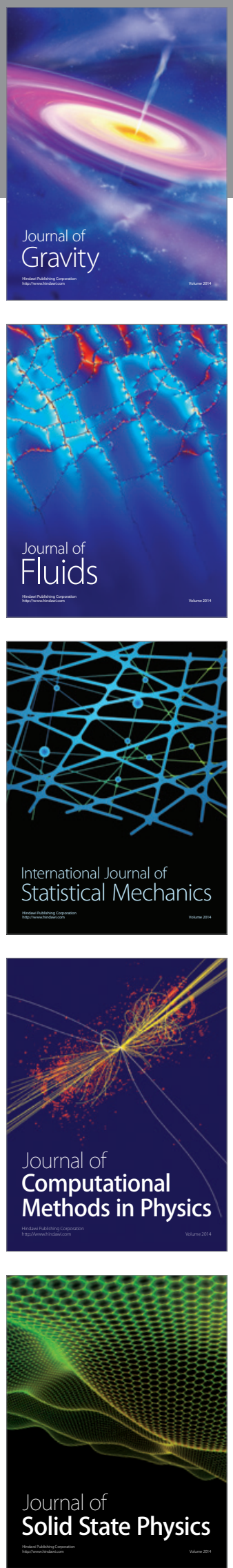

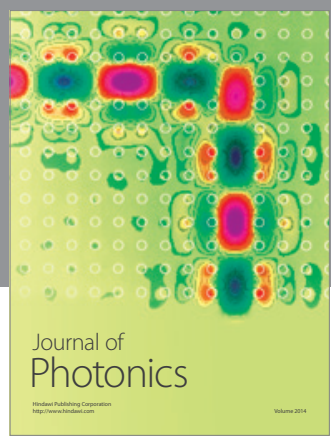

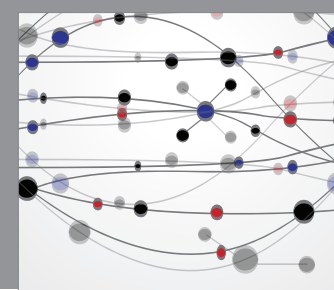

The Scientific World Journal

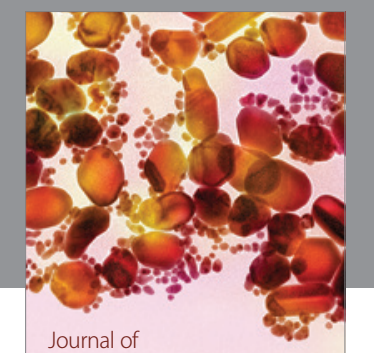

Soft Matter
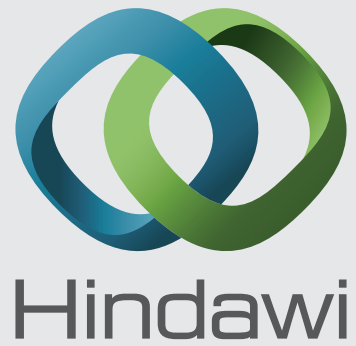

Submit your manuscripts at

http://www.hindawi.com
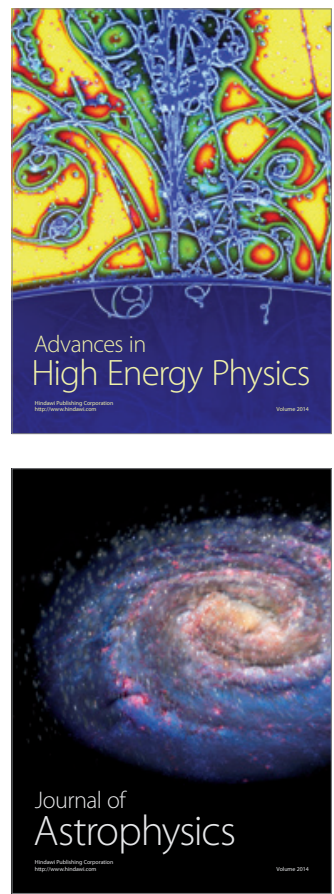
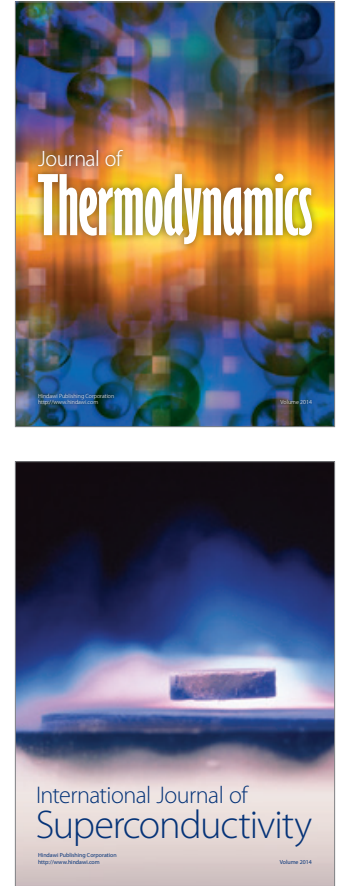
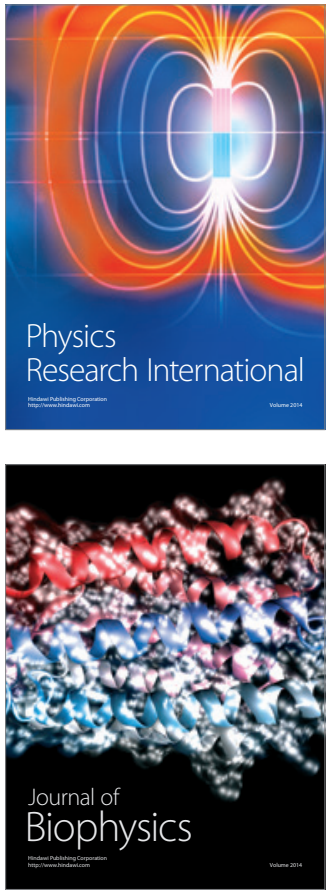
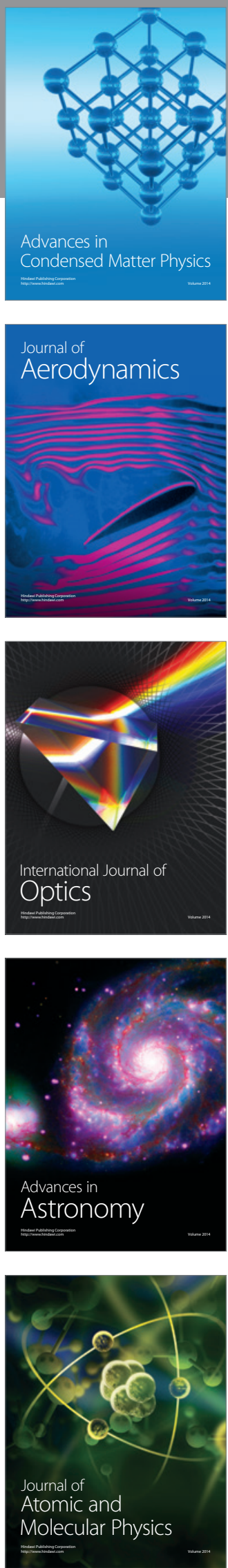